\section{PROTOCOLO dE INJEÇÕES INTRAVÍtREAS do Hospital \\ de Clínicas de Porto Alegre: uma ReVisão de \\ CONDUTAS PARA UMA ASSISTÊNCIA MAIS QUALIFICADA}

Hospital de Clínicas de Porto Alegre intravitreal

INJECTION PROTOCOL: A LITERATURE REVIEW TO

IMPROVE ASSISTANCE

Lúcio Falavigna ${ }^{1}$, Karla Scheid ${ }^{1}$, Daniel Lavinsky ${ }^{1,2}$, Felipe Mallmann ${ }^{1}$

\section{RESUMO}

A injeção intravítrea é o procedimento oftalmológico mais realizado no mundo. Através dessa técnica é possível administrar medicamentos para o segmento posterior do olho, sendo a via de escolha para o tratamento da maioria das patologias coriorretinianas. O evento ocular adverso mais grave é a endoftalmite pós injeção. Diversas medidas foram sugeridas para diminuição do risco dessa complicação, com evidências variáveis. Tendo em vista o grande número de injeções realizado em nosso serviço de oftalmologia do Hospital de Clínicas de Porto Alegre (HCPA), realizamos uma revisão da literatura com o objetivo de definir as melhores práticas. Nós descrevemos a evidência disponível para os principais aspectos dos diferentes estágios do procedimento (pré injeção, peri injeção e pós injeção) e apresentamos nosso protocolo. A medida com maior evidência científica na profilaxia da endoftalmite é a aplicação de iodopovidona tópica pelo menos trinta segundos antes da injeção.

Palavras-chave: Injeção intravítrea; anti-VEGF; endoftalmite pós injeção

\section{ABSTRACT}

Intravitreal injection is the most widely performed ophthalmologic procedure in the world. This technique allows for delivering therapeutic substances to the posterior segment of the eye, being the route of choice for the treatment of most chorioretinal diseases. The most feared adverse event is post-injection endophthalmitis. Many measures have been described to lower the risk of this complication, with variable evidence. In view of the great number of injections performed at our ophthalmology practice at Hospital de Clínicas de Porto Alegre (HCPA), we searched the literature for available evidence on the best practice standards. We describe the available evidence for major aspects of the different stages of the procedure (pre-injection, peri-injection, and post-injection) and then introduce our protocol. The measure with most scientific evidence of efficacy in preventing endophthalmitis is topical povidoneiodine administration at least 30 seconds before the injection.

Keywords: Intravitreal injection; anti-VEGF; post-injection endophthalmitis

\section{INTRODUÇÃO}

A injeção intravítrea é o procedimento oftalmológico mais realizado no mundo. Nos Estados Unidos foi evidenciado um aumento de 2.943 indicações no ano 2000 para 2.619.950 em 2014. Com estudos demonstrando a eficácia dos agentes anti-fator de crescimento vascular endotelial (anti-vascular endothelial growth factor; anti-VEGF) para o tratamento de diversas patologias retinianas de elevada prevalência e morbidade, sua indicação aumentou significativamente na última década ${ }^{1,2}$.
Clin Biomed Res. 2020;40(3):173-183

1 Serviço de Oftalmologia do Hospital de Clínicas de Porto Alegre. Porto Alegre, Rio Grande do Sul, Brasil.

2 Faculdade de Medicina, Universidade Federal do Rio Grande do Sul. Porto Alegre, RS, Brasil.

Corresponding author: Felipe Mallmann felipemallmann@hcpa.edu.br Hospital de Clínicas de Porto Alegre Rua Ramiro Barcelos, 2350. 90035903, Porto Alegre, RS, Brasil. 
Entre essas patologias destacam-se o edema macular diabético (EMD), o edema macular associado com oclusão venosa e a degeneração macular relacionada com a idade forma neovascular (DMRI). É considerada a via de administração preferencial para o tratamento da maioria das patologias coriorretinianas, visto que permite a obtenção de concentrações adequadas nesses tecidos com baixa taxa de efeitos adversos sistêmicos ${ }^{3}$.

A presença das barreiras hematorretinianas limita a absorção de substâncias a partir da circulação sistêmica e, consequentemente, não são obtidas concentrações adequadas na cavidade vítrea ${ }^{3}$. Diversas doenças que apresentam edema macular como manifestação têm como principal fator fisiopatológico o aumento da permeabilidade das barreiras hematorretinianas, com consequente extravasamento de líquido para o espaço retiniano extracelular. Frequentemente estão implicados nesse processo o aumento dos níveis de VEGF e das citocinas pró-inflamatórias, que desorganizam as estruturas proteicas relacionadas com a integridade da barreira hematorretiniana ${ }^{4-6}$.

$\mathrm{O}$ uso de anti-VEGF e/ou corticosteroides intravítreos, portanto, mostraram-se eficazes no tratamento do edema macular secundário a diversas patologias $^{7-9}$. Entre os anti-VEGF atualmente em uso no Brasil temos o bevacizumabe (Avastin; Genentech [São Francisco, Califórnia]), ranibizumabe (Lucentis; Genentech [São Francisco, Califórnia]) e aflibercepte (Eylia; Regeneron Pharmaceuticals Inc., [Renssealer, Nova York]). Cabe ressaltar que o uso do bevacizumabe na terapêutica ocular é considerado off-label. Já dentre os corticosteróides, temos disponíveis o acetato de triancinolona (Ophtaac 40, Ophthalmos S/A [São Paulo, Brasil]) e o implante de dexametasona (Ozurdex; Allergan Pharmaceuticals [Irlanda]).

Outras classes de agentes terapêuticos administradas através das injeções intravítreas são os antibióticos, utilizados no tratamento das endoftalmites, gases, para o manejo de casos específicos de descolamento de retina e quimioterápios, na terapêutica de neoplasias intraoculares ${ }^{10,11}$. Atualmente são realizadas uma média anual de 1.200 injeções intravítreas no HCPA, configurando-a como nosso procedimento de maior volume.

Entre as potenciais complicações, temos particular preocupação com a endoftalmite infecciosa, tendo em vista sua elevada morbidade ocular, com risco de cegueira e até de perda do globo ocular. A incidência dessa complicação varia na literatura de $0.028 \%$ a $0.056 \%{ }^{12,13}$. Outros eventos adversos relatados incluem a ceratoconjuntivite química, hiposfagma, catarata, glaucoma, hemorragias intraoculares, descolamento de retina e endoftalmite estéril.

Pelo elevado volume do procedimento em nosso serviço e com o intuito de limitar a incidência de complicações, revisamos a literatura com vistas a identificar as melhores práticas e implementá-las em nossa prática assistencial. Para tanto, são relevantes na adequada realização do procedimento aspectos do pré operatório, como identificação de patologias oculares e sistêmicas pré existentes que aumentem o risco de eventos adversos. Durante o perioperatório, cuidados associados com a esterilidade e conforto do procedimento. Finalmente, no pós operatório, a identificação e manejo precoce de complicações potencialmente cegantes é fundamental.

\section{AVALIAÇÃO PRÉ-OPERATÓRIA}

Não há contraindicações absolutas para a realização do procedimento, porém a relação risco $\mathrm{x}$ benefício deve sempre ser ponderada ${ }^{14}$. Os pacientes devem ser avaliados quanto à presença de alterações oculares e sistêmicas que podem aumentar o risco de eventos adversos. Destacam-se a presença de glaucoma, alterações de superfície ocular, cirurgias oculares prévias, gestação e doenças cardiovasculares, especialmente se há relato de descompensação recente.

\section{Condições oculares}

\section{Alterações de superfície ocular}

Qualquer alteração de superfície ocular pode potencialmente aumentar o risco de endoftalmite, devendo sempre ser considerado o risco $x$ benefício. Infecções ativas palpebrais ou de superfície ocular - blefarite, hordéolo, conjuntivite, dacriocistite, dacrioadenite devem ser tratadas adequadamente antes do procedimento, evidências indicam aumento do risco de endoftalmite se realizada a injeção nesse contexto ${ }^{14-16}$. $O$ procedimento pode ser realizado em pacientes com blefarite crônica em tratamento ${ }^{15}$.

Em nosso serviço, quando evidenciado processo infeccioso ocular ativo, realizamos o tratamento da patologia antes de indicar a injeção. No infortúnio de tal alteração ser suspeitada no bloco cirúrgico, o paciente é instruído a comparecer no ambulatório para avaliação e caso confirmado, o tratamento é instituído e a injeção adiada.

\section{Hipertensão ocular e glaucoma}

$\mathrm{O}$ aumento de volume associado com a injeção intravítrea $(0,05$ a $0,1 \mathrm{ml})$ frequentemente induz episódios de hipertensão ocular imediatos, sendo relatadas pressões intraoculares (PIO) maiores que $30 \mathrm{mmHg}$ em até $30 \%$ dos procedimentos após 5 minutos da injeção ${ }^{17}$. Esses picos tendem a resolver espontaneamente em 30 minutos na maioria dos casos, podendo se estender em portadores de glaucoma ou de hipertensão ocular ${ }^{18-20}$. 
Há evidências de que o olho saudável pode tolerar episódios hipertensivos sem sequelas a longo prazo, porém em pacientes com nervo óptico vulnerável incluindo glaucomatosos e naqueles com fatores de risco para neuropatia óptica isquêmica ou oclusões vasculares - os picos hipertensivos, isolados e/ou cumulativamente ao longo de múltiplas injeções, podem resultar em sequelas visuais ${ }^{21}$.

Há evidências conflitantes quanto ao uso de colírios anti-hipertensivos na profilaxia dos picos hipertensivos imediatos ${ }^{22-24}$. Em pacientes com histórico de pico hipertensivo significativo prévio ou em olhos de maior risco, pode ser considerada a massagem do globo ocular antes do procedimento, evitando-se a manipulação das pálpebras ${ }^{14}$. Caso o paciente apresente pico hipertensivo agudo sintomático, com dor e piora visual, pode ser realizada paracentese na câmara anterior para alívio imediato ${ }^{15}$.

$\mathrm{O}$ aumento da PIO também pode ocorrer de forma persistente, secundário às propriedades dos medicamentos administrados. Os corticoides são associados mais frequentemente com essa complicação, relatada em 30-60\% das injeções com acetato de triancinolona ${ }^{25}$. Os agentes anti-VEGF também foram implicados nesse efeito adverso, com taxas de até $11.6 \%^{26}$. Na maioria dos casos, a hipertensão é transitória e o controle adequado da PIO pode ser obtido com tratamento tópico ${ }^{17,27}$.

O risco cumulativo de múltiplos episódios de hipertensão imediata ou persistente deve ser levado em consideração, principalmente em pacientes suscetíveis ao dano. Idealmente, esses pacientes devem estar com suas patologias de base compensadas antes do procedimento e realizado um acompanhamento adequado no sentido de identificar precocemente a necessidade de intervenção. Não realizamos a massagem do globo ocular de forma rotineira no transoperatório.

Em geral, contraindicamos injeção intravítrea de corticoides nos portadores de glaucoma avançado ou de difícil controle, sendo os demais casos avaliados individualmente quanto ao perfil risco $\mathrm{x}$ benefício.

\section{Cirurgias oculares prévias}

Não há cirurgia ocular prévia que constitua uma contraindicação para o procedimento ${ }^{14,15}$. A injeção pode ser administrada com segurança em 360 graus ao redor do limbo, devendo-se evitar locais de conjuntiva ou pars plana alteradas, como bolhas filtrantes pós trabeculectomia, esclerotomias recentes ou cistos de pars plana. Cirurgia recente de catarata, realizada nos últimos 3 meses, não é uma contraindicação ao procedimento ${ }^{14}$.

\section{Condições sistêmicas}

\section{Doenças sistêmicas}

O uso sistêmico de bevacizumabe (BEZ) para o tratamento de câncer colorretal foi associado com complicações cardiovasculares ${ }^{28,29}$. Em virtude da terem sido detectadas concentrações sistêmicas desses fármacos após administração intraocular, há uma preocupação com os potenciais riscos sistêmicos ${ }^{30}$.

Evidências sugerem que o uso de anti-VEGF intraocular não aumenta o risco de eventos adversos sistêmicos de forma significativa. O uso mensal em comparação com protocolos de injeção menos frequentes também não demonstrou aumento no risco. Os 3 agentes disponíveis (ranibizumabe, aflibercept e BEZ) foram comparáveis entre si nesse quesito. O uso de ranibizumabe (RAZ) para tratamento de DMRI em pacientes mais idosos pode estar associado com aumento do risco de eventos hemorrágicos não oculares ${ }^{31}$. Não está indicada a suspensão de anti-coagulantes antes do procedimento, visto que a sua manutenção não foi associada com aumento no risco de eventos adversos hemorrágicos ${ }^{32}$.

Apesar da evidência disponível, é recomendado avaliar o risco $\mathrm{x}$ benefício desses medicamentos com a equipe assistencial em pacientes com quadros sistêmicos complexos, principalmente se histórico de eventos agudos recentes ${ }^{4}$. A administração intravítrea de acetato de triancinolona não está associada com riscos significativos de efeitos adversos sistêmicos ${ }^{33}$.

\section{Gestação e amamentação}

Apesar do grande volume de injeções intravítreas realizadas anualmente, há pouca evidência no uso dessas substâncias durante a gestação e amamentação. Há relatos de uso de triancinolona intraocular durante a gestação sem intercorrências ${ }^{34}$. A preocupação é maior com uso dos agentes anti-VEGF pelas potenciais alterações no desenvolvimento placentário e do feto, principalmente no início da gestação.

Apesar de um relato de perda gestacional com uso de $B E Z^{35}$ outros autores utilizaram o medicamento, inclusive no primeiro trimestre, sem intercorrências gestacionais $^{35,36}$. Um estudo em pacientes recebendo BEZ mensal durante período de amamentação não evidenciou níveis detectáveis do fármaco no leite materno ${ }^{37}$. Entretanto, não há estudos avaliando os efeitos a longo prazo dessas substâncias no desenvolvimento dessas crianças.

O risco-benefício do uso de esteróides intraoculares parece aceitável durante a gestação, porém o uso de anti-VEGF deve ser avaliado mais cuidadosamente, levando em conta os potenciais riscos gestacionais. Não parece haver contra-indicação para a administração desses medicamentos durante a amamentação ${ }^{15}$. 
Falavigna et al.

\section{AVALIAÇÃO E CONDUTAS PERIOPERATÓRIAS}

Os cuidados no peri e transoperatório visam a realização de um procedimento estéril com baixo risco de complicações, de forma confortável e segura para o paciente. A endoftalmite é uma complicação rara, porém de elevada morbidade ocular. A incidência dessa complicação varia na literatura de $0.028 \%$ a $0.056 \%{ }^{12,13}$. Em nossa instituição a taxa dessa complicação é de $0.03 \%$ até o momento da confecção desse artigo (dados não publicados), semelhante aos índices mais favoráveis relatados na literatura.

Diversas medidas são relatadas para diminuir do risco dessa complicação, com evidências de qualidade variável. Poucos estudos prospectivos foram realizados em função da baixa incidência dessa complicação e da complexidade inerente às diversas variáveis que deveriam ser avaliadas. Em função disso, há grande heterogeneidade nas práticas entre os serviços de oftalmologia ${ }^{38}$.

\section{Ambiente do procedimento}

Há o relato de injeções intravítreas sendo realizadas em consultório médico, em salas adaptadas para o procedimento e em salas cirúrgicas convencionais. Nos EUA e Canadá, a maioria das injeções é realizada em consultório médico ${ }^{39}$.

Um estudo retrospectivo avaliando 500.000 injeções realizadas em consultório médico demonstrou uma taxa de endoftalmite de $0.036 \%{ }^{40}$. Outros estudos retrospectivos avaliando 5.429 e 40.000 injeções realizadas no bloco cirúrgico, evidenciaram taxas de $0.09 \%$ e $0.007 \%$, respectivamente ${ }^{41,42}$. Resultados combinados de séries de injeções realizadas no bloco cirúrgico evidenciaram taxas de $0.0076 \%{ }^{13}$.

A literatura aponta que as taxas de endoftalmite são muito baixas em ambos os contextos e até comparáveis em algumas séries de casos, porém não há estudos prospectivos randomizados comparandoos diretamente. Os três locais são considerados adequados para realizar o procedimento ${ }^{14,15}$. Em nosso hospital, em virtude de normativas e regulamentações internas e externas, realizamos o procedimento em sala cirúrgica.

\section{Uso de máscara e restrição à conversa}

Em metanálise avaliando casos de endoftalmite pós injeção intravítrea, foi identificada uma prevalência três vezes maior de Streptococcus ssp. como agente responsável em relação aos casos pós cirurgia de catarata, em que geralmente é feito o uso de máscaras ${ }^{43}$. O Streptococcus ssp. é componente usual da flora orofaríngea, sugerindo que possa haver uma maior contaminação a partir de gotículas nesses casos. Estudo simulando o cenário do procedimento demonstrou haver um maior crescimento bacteriano em placas de cultura dispostas próximas do cirurgião após períodos de conversa sem o uso de máscaras em relação a um contexto com máscaras ou mantendo-se o silêncio ${ }^{44}$.

Em 2004 foi investigado um surto de meningite pós mielografia associada com microorganismos da flora orofaríngea. Foi evidenciada relação com o fato de os médicos não usarem máscaras e recomendado o uso rotineiro de máscara cirúrgica em procedimentos envolvendo administração de substâncias no espaço subdural ou o canal medular ${ }^{45}$. Cabe a comparação entre a situação descrita previamente com as injeções intravítreas, em que medicamentos são administrados em um espaço biológico estéril cuja contaminação tem repercussões potencialmente graves.

Estudo retrospectivo de séries de casos demonstrou uma redução do risco de endoftalmite infecciosa com uma política de restrição à conversa durante o procedimento. Além disso, dentre os casos no grupo sem conversa, o isolamento de microorganismos associados com a mucosa oral foi significativamente menor ${ }^{46}$. Apesar de não haver estudos prospectivos adequados avaliando essas intervenções, é considerado adequado o uso de máscaras e realizar restrição à conversa durante o procedimento, tendo em vista o baixo custo e os potenciais benefícios ${ }^{14,15,30}$.

\section{Higiene das mãos e uso de luvas estéreis}

O uso de luvas e a realização de higiene das mãos é consistente com a realização de procedimentos médicos em geral. As luvas protegem o médico do contato com secreções do paciente e protegem o paciente de contaminação através das mãos do médico. Contudo, não há evidências demonstrando que o uso de luvas diminua o risco de endoftalmite.

Estudo retrospectivo em um único centro, avaliando 154.198 injeções, não identificou o uso de luvas como preditor estatisticamente significativo de endoftalmite ${ }^{47}$. Em 2 estudos prospectivos, controlados, randomizados em que não foi feito o uso de luvas, a taxa de endoftalmite foi de $0.078 \%$ após 3.383 injeções $^{48}$.

Em nossa instituição, indicamos a higiene das mãos imediatamente antes de cada procedimento, assim como o uso de luvas estéreis.

\section{Desinfecção da pele periocular}

Desinfecção da pele periocular com iodopovidona $10 \%$ é procedimento padrão antes da realização de diversos procedimentos oftalmológicos incisionais. Não há evidências fortes para embasar o seu uso antes da injeção intravítrea. Quando realizada, devese evitar a manipulação excessiva das pálpebras a fim de evitar o aumento da contaminação conjuntival por secreções das glândulas palpebrais ${ }^{15}$.

Apesar de não termos evidências consistentes da eficácia deste método para o procedimento de 
injeção intravítrea, ainda realizamos a assepsia da pele por uma questão de risco-benefício.

\section{Campo cirúrgico estéril adesivo}

O uso de campo cirúrgico estéril adesivo tem como objetivo manter a esterilidade do local do procedimento $\mathrm{e}$ isolar potenciais focos contaminantes como a região nasal, vias respiratórias e cabelo.

Não há evidências que sugiram que o uso desse recurso diminua de forma significativa o risco de endoftalmite ${ }^{14,15,38}$. Além disso, pacientes relataram que após a aplicação da injeção, o campo cirúrgico estéril adesivo é o segundo aspecto mais desconfortável do procedimento ${ }^{49}$. Em nossa instituição, fazemos uso rotineiro de campos cirúrgicos estéreis não adesivos.

\section{Blefarostato}

Uma das fontes de contaminação para a ocorrência de endoftalmite é a margem palpebral, podendo a mesma contaminar a agulha ou diretamente o local onde é realizada a injeção. O uso do blefarostato permite afastar as margens palpebrais do sítio da injeção e estabiliza as pálpebras para diminuir o risco de haver toque na agulha.

Um estudo randomizado controlado duplo cego avaliando 1186 pacientes com DMRI evidenciou taxas mais elevadas de endoftalmite sem o uso de blefarostato ${ }^{50}$. Em contrapartida, um estudo retrospectivo avaliando 27.736 injeções não encontrou diferença significativa com e sem o uso de blefarostato ${ }^{51}$. Outros métodos para afastar as pálpebras foram descritos, como a retração manual, havendo relato de taxa de endoftalmite de $0.0015 \%$ em um estudo retrospectivo com 78.009 injeções ${ }^{52}$.

Apesar de haver controvérsia no assunto, é considerado adequado que algum método seja empregado para o afastamento das pálpebras ${ }^{14,15,38}$. Em nosso hospital, preconizamos o uso, pois acreditamos que facilita a aplicação e minimiza o risco pelo contato da margem palpebral com a agulha.

\section{Anestesia e analgesia}

Diversas modalidades de anestesia estão disponíveis para a realização da injeção intravítrea, incluindo tópica com colírio, tópica com gel e subconjuntival. Há maior preferência pela anestesia tópica por parte dos retinólogos ${ }^{53}$. Atualmente, não dispomos de evidências para priorizar uma modalidade específica de anestesia em detrimento das demais, porém sugere-se o uso da anestesia tópica em virtude de ser menos invasiva ${ }^{15}$.

Há uma preocupação com o uso da anestesia tópica com gel pelo risco de reduzir o contato do antisséptico com a superfície ocular. Em experimentos com placas de agar, o uso de lidocaína gel diminuiu a eficácia da iodopovidona 5\% tópica (PVPI) em erradicar os microorganismos, possivelmente por criar um efeito de barreira. No entanto, quando o antisséptico é aplicado antes do gel, não há redução da eficácia in vitro ${ }^{54}$. Caso optado por essa forma de anestésico, deve-se atentar para o uso do PVPI antes da aplicação do gel. Em nosso serviço, realizamos o processo anestésico com proximetacaína colírio.

\section{Antissepsia tópica}

Contaminação do local da injeção pode ocorrer através de patógenos da conjuntiva e margem palpebral do paciente, assim como por contaminantes da mucosa oral do médico ao conversar durante o procedimento ${ }^{44,55}$.

A iodopovidona $5 \%$ tópica (PVPI) é o padrão para redução de patógenos da superfície ocular antes de procedimentos oftalmológicos incisionais. Extrapolando dados referentes às cirurgias de catarata, foi a única intervenção considerada como grau de evidência 2 para profilaxia de endoftalmite pós operatória ${ }^{56}$. Ela possui um amplo espectro de ação antimicrobiana, sem relatos de resistência bacteriana adquirida ${ }^{57}$.

A aplicação do PVPI pelo menos 30 segundos antes da injeção é considerado adequado para reduzir significativamente as contagens bacterianas da superfície ocular e uma segunda aplicação traz benefícios adicionais ${ }^{58,59}$. O uso do PVPI após a colocação do blefarostato parece diminuir a incidência de endoftalmite ${ }^{60}$ e deve ser o último colírio aplicado antes da injeção ${ }^{14}$.

A alegação de alergia ao iodo é um impeditivo frequente ao uso do PVPI. Os pacientes podem apresentar episódios de conjuntivite e ceratite tóxicas que frequentemente são confundidos com alergia ${ }^{61}$. Não está descrito na literatura anafilaxia após instilação de PVPI ocular e quadros alérgicos verdadeiros são considerados raros $^{62,63}$. Alergia ao contraste iodado e a frutos do mar são desencadeados por substâncias não relacionadas aos componentes do PVPI e, portanto, não representam contraindicação ao seu uso ${ }^{64,65}$. Em pacientes não tolerantes ao PVPI tópico, o gluconato de clorexidina aquosa $0.1 \%$ pode ser usado como alternativa segura e com taxas comparáveis de endoftalmite ${ }^{13,38,66}$.

Na nossa rotina atual, é realizada a aplicação de PVPI 5\% tópico uma única vez quando o paciente entra na sala cirúrgica, após a anestesia tópica, respeitando um período de pelo menos 3 minutos até aplicar a injeção.

\section{Técnica}

O uso de uma agulha de calibre e comprimento adequados é importante para garantir conforto e segurança ao procedimento. Há evidências de que o calibre de 30 gauge ou mais fino está relacionado a um maior conforto para o paciente, assim como menor risco de refluxo vítreo na incisão ${ }^{67-69}$. É considerado o calibre ideal pelos principais guidelines ${ }^{14,15}$ 
Falavigna et al.

e recomendado na bula dos principais medicamentos intravítreos administrados na prática oftalmológica.

O comprimento da agulha deve ser o suficiente para transfixar a pars plana sem aumentar significativamente o risco de lesão às estruturas intraoculares, geralmente entre 13 e $18 \mathrm{~mm}$ é considerado adequado. A injeção é tipicamente realizada a $3.5-4.0 \mathrm{~mm}$ do limbo, distância que acaba coincidindo com a região da pars plana. Injeções mais anteriores aumentam o risco de catarata traumática e lesão da pars plicata, mais posteriores aumentam o risco de rotura retiniana.

Qualquer quadrante é considerado adequado, preferindo-se realizar entre os músculos retos ${ }^{14,15}$. Estudo de Karimi et al. ${ }^{70}$, verificou que a dor durante o procedimento foi crescente nos respectivos quadrantes: nasal superior, temporal inferior, nasal inferior e temporal superior. Entretanto, a margem de segurança teórica é menor nos quadrantes nasais, principalmente em pacientes com menor comprimento axial. Portanto, optamos pela rotina de injeções temporais inferiores.

Deve-se evitar locais de superfície ocular e pars plana alteradas, conforme discutido previamente na seção de avaliação pré-operatória. Não há necessidade de tunelizar a incisão, devendo-se inserir pelo menos $6 \mathrm{~mm}$ (cerca de 2/3 do comprimento da maioria das agulhas) direcionada para o polo posterior do olho, evitando-se o toque da agulha na margem palpebral ${ }^{14,15}$. Injeção abrupta do medicamento é desestimulada, pois pode aumentar o risco de hipertensão ocular imediata ${ }^{21}$.

\section{Antibióticos tópicos perioperatórios}

O uso de antibióticos no perioperatório das injecões intravítreas era prática comum, embasada na teoria de que a diminuição da carga patogênica na superfície ocular diminuiria o risco de endoftalmite.

Uma metanálise avaliando 174.000 injeções concluiu que não há benefício no uso de antibióticos pré e pós operatórios como forma de profilaxia ${ }^{71}$. Além disso, alguns estudos demonstraram aumento nas taxas de endoftalmite com o uso rotineiro de antibióticos nesse contexto, sendo atribuído à indução de resistência bacteriana na superfície ocular ${ }^{72,73}$. Portanto, a evidência atual não embasa o uso de antibióticos como forma de profilaxia para endoftalmite pós injeção intravítrea ${ }^{14,15,38}$.

Em nossa instituição, não fazemos uso rotineiro de antibióticos fora do período transoperatório. Apesar de não haver evidências significativas embasando o uso de antibiótico tópico logo ao término da injeção intravítrea, realizamos aplicação de uma gota de ofloxacina colírio ou PVPI 5\% antes da retirada do blefarostato, tendo em vista que a relação risco $\mathrm{x}$ benefício parece razoável.

\section{Medicamentos manipulados}

Apesar dos medicamentos ranibizumabe e aflibercepte serem os fármacos aprovados em bula para uso intraocular, eles não estão disponíveis de rotina e o $B E Z$, em seu uso off-label é o agente anti-VEGF predominantemente utilizado na nossa instituição. Tipicamente é fracionado em diversas seringas a partir de um frasco estéril, tornando o custo muito menor em relação aos demais fármacos dessa classe. Contudo, há uma preocupação quanto ao risco de contaminação no processo. Há relatos de surtos de endoftalmite associados à manipulação do $B E Z^{74}$, porém fora desse contexto não há evidências significativas do aumento do risco de infecção intraocular.

Um estudo retrospectivo multicêntrico avaliando 503.890 injeções não identificou diferenças significativas entre as taxas de endoftalmite infecciosa entre o uso de BEZ fracionado, RAZ e aflibercepte ${ }^{40}$. Tipicamente, com a realização do fracionamento em ambiente estéril e com os cuidados adequados, o medicamento não apresenta contaminantes ou endotoxinas ${ }^{75}$.

Em nossa instituição, os fármacos utilizados com maior frequência são o BEZ e o acetato de triancinolona. O primeiro, conforme mencionado anteriormente, necessita de manipulação para fracionar a ampola em diversas seringas estéreis com a dose adequada. Esse processo é realizado por um departamento específico da nossa instituição, a central de misturas intravenosas (CMIV), que conta profissionais capacitados e segue as regulamentações vigentes para manuseio destes fármacos.

Condizente com os dados da literatura, na trajetória de 10 anos em que realizamos aproximadamente 10.000 injeções de BEZ, tivemos uma incidência de 3 casos de endoftalmite em períodos distintos (dados não publicados), o que reforça a segurança do processo.

\section{Injeções bilaterais no mesmo dia}

O edema macular diabético e a degeneração macular relacionada com a idade constituem $80 \%$ das indicações de injeção intravítreas em nosso serviço, com elevada taxa de bilateralidade, sendo esse último particularmente associado com idades mais avançadas. Tendo em vista o grande fardo de injeções e consultas nessa população, diversos serviços adotam a prática de realizar injeções bilaterais no mesmo dia ${ }^{73}$, com o intuito de melhorar a aderência e conforto para o paciente ${ }^{75}$, assim como custo-efetividade.

Apesar de haver relatos na literatura de endoftalmite bilateral após injeções nesse contexto ${ }^{73}$, a incidência parece ser muito baixa. Em revisão de 101.932 injeções bilaterais, ambos os olhos no mesmo dia, a taxa de endoftalmite foi de $0.027 \%$, sem casos com acometimento bilateral ${ }^{76}$. 
A ponderação risco $\mathrm{x}$ benefício deve ser discutida com o paciente. Deve-se considerar cada injeção como um procedimento individual, utilizando instrumental separado para cada olho, troca de paramentação e preferencialmente evitar o uso de medicamentos do mesmo lote ${ }^{14,15}$. Atualmente, não realizamos injeções bilaterais no mesmo dia no HCPA.

\section{PÓS OPERATÓRIO}

Não há estudos avaliando as medidas pós operatórias, sendo a evidência formada principalmente a partir de consenso de especialistas.

\section{Manejo pós-injeção}

É sugerido a realização de um teste rudimentar de acuidade visual logo após o término do procedimento, com teste de contagem de dedos, para se certificar que há perfusão da artéria central da retina, e a medida da pressão intraocular com tonômetro de aplanação ou palpação bidigital antes de liberar o paciente da sala cirúrgica ${ }^{14,15}$. Conforme discutido previamente, picos hipertensivos imediatos sintomáticos, com dor e piora visual, podem ser tratados com paracentese da câmara anterior.

O paciente deve receber orientação quanto aos sinais de alarme para as principais complicações do pós operatório e informações quanto às portas de entrada do serviço de saúde caso venha a apresentá-los. O retorno ambulatorial é estipulado conforme o acompanhamento da patologia de base e do medicamento administrado.

Os principais sintomas que devem ser enfatizados para os pacientes são a dor ocular ou piora gradual do desconforto, redução da visão, aumento da sensibilidade a luz e piora da hiperemia ocular. A observação de moscas volantes após a injeção é algo usual e dentro da normalidade. Deve evitar a manipulação ocular nas primeiros dias após o procedimento ${ }^{14,15}$.

\section{Complicações}

Apesar da baixa absorção sistêmica, há a preocupação de agentes anti-VEGF intraoculares aumentarem o risco de eventos cardiovasculares, principalmente em populações idosas ou apresentando doenças isquêmicas e diabetes mellitus. A maioria dos trabalhos não demonstrou um aumento significativo do risco de eventos adversos sistêmicos (EAS) em pacientes utilizando medicamentos dentro da cavidade vítrea ${ }^{31}$.

Já os eventos adversos oculares (EAO) são mais frequentes, podendo estar relacionados ao procedimento em si ou ao medicamento utilizado. É imprescindível a identificação precoce dessas situações para adequado manejo.,

\section{Superfície ocular}

A hiperemia e hemorragia subconjuntivais são os EAO mais comuns, geralmente secundários ao trauma cirúrgico e presentes logo após o procedimento, costumam resolver ao longo de poucos dias sem sequelas ${ }^{77}$. Ceratoconjuntivite química e defeitos epiteliais corneanos podem ser secundários aos medicamentos tópicos utilizados no perioperatório, muitas vezes causando grande desconforto no pósoperatório imediato ${ }^{77}$. O uso de lubrificantes oculares sem conservante pode ser indicado nesses casos, na posologia de 1 gota a cada 2 horas, para amenizar os sintomas que tendem a resolver em 24 a 48 horas. Entretanto, na presença de dor forte associada com fotofobia, uma avaliação presencial é necessária para a realização do diagnóstico de defeito epitelial e tratamento adequado com oclusão ou uso de lente de contato terapêutica, conforme a gravidade.

\section{Hipertensão ocular}

Tópico abordado de forma mais extensa no item "hipertensão ocular e glaucoma". A hipertensão ocular sustentada é mais frequente com o uso de corticóides intravítreos, ocorrendo em cerca de $30 \%$ dos $\operatorname{casos}^{25}$. A evolução para glaucoma de difícil controle, necessitando de cirurgia é incomum ${ }^{27}$.

Em nosso serviço, os pacientes são acompanhados no pós operatório com aferição periódica da PIO, sendo instituído tratamento hipotensor nos casos em que essa se mostra significativamente elevada, especialmente nos portadores de fatores de risco para dano glaucomatoso. Tendo em vista a natureza transitória da hipertensão ocular nesse contexto, costuma ser possível a posterior suspensão do tratamento na maioria dos pacientes.

\section{Catarata}

A indução de catarata pode ser traumática em virtude de um toque da agulha durante o procedimento, ou ser secundária ao efeito adverso do medicamento. No primeiro caso, há ruptura da cápsula posterior do cristalino em boa parte dos casos, implicando uma intervenção mais complexa, onde pode haver deslocamento de material cristaliniano para a cavidade vítrea, sendo um EA infrequente.

Já a incidência de catarata significativa induzida pelo efeito da corticoterapia intraocular, pode variar conforme a concentração da droga utilizada. Durante o acompanhamento de 3 anos de pacientes com EMD, $83 \%$ do grupo que recebeu $4 \mathrm{mg}$ de triancinolona e $46 \%$ que recebeu $1 \mathrm{mg}$ necessitaram de cirurgia de catarata ${ }^{27}$. Já os anti-VEGFs não parecem aumentar o risco de catarata de forma significativa ${ }^{78}$.

\section{Complicações vitreorretinianas}

A hemorragia vítrea é um EAO incomum, podendo ocorrer por trauma das estruturas ciliares durante 
o procedimento ou por uma alteração da superfície vítreo-retiniana secundária à patologia de base ${ }^{79,80}$. Inflamações intraoculares e endoftalmite estéril secundárias aos componentes do fármaco já foram relatadas, mas são pouco frequentes, principalmente com o uso de medicamentos anti-VEGF ${ }^{81}$.

Finalmente, os EAO que mais geram morbidade são a endoftalmite infecciosa e o descolamento de retina, ocorrendo em menos de $1 \%$ dos casos em praticamente todos os estudos e metanálises sobre o procedimento ${ }^{12,13,78}$. A presença destas complicações deve ser reconhecida com brevidade e tratada através de procedimentos vítreo-retinianos para que não haja comprometimento do prognóstico visual.

\section{DISCUSSÃO}

Após extensa revisão da literatura, elaboramos um protocolo visando a instituição das melhores práticas atuais em nosso serviço. No geral, as medidas que adotamos (Tabela 1) estão de acordo com os consensos.

Tabela 1: Descrição do procedimento conforme medidas estipuladas no HCPA.

\begin{tabular}{cl}
\hline \multicolumn{2}{l}{ Técnica de Injeção Intravítrea estipulada no HCPA } \\
\hline $\mathbf{1}$ & $\begin{array}{l}\text { Instilação de anestésico local } \\
\text { (proximetacaína colírio) } 01 \text { gota }\end{array}$ \\
\hline $\mathbf{2}$ & Instilação de 1 gota de PVPI 5\% \\
\hline $\mathbf{3}$ & Antissepsia das mãos e colocação de luvas estéreis \\
\hline $\mathbf{4}$ & $\begin{array}{l}\text { Antissepsia da pele com gazes umedecidas em } \\
\text { iodopovidona 10\% }\end{array}$ \\
\hline $\mathbf{5}$ & Colocação de campo oftálmico fenestrado estéril \\
\hline $\mathbf{6}$ & Colocação de blefarostato de Barraquer \\
\hline $\mathbf{7}$ & $\begin{array}{l}\text { Instilação de anestésico local } \\
\text { (proximetacaína colírio) 01 gota }\end{array}$ \\
\hline $\mathbf{8}$ & Instilação de 1 gota de PVPI 5\% \\
\hline $\mathbf{9}$ & Marcação de local de injeção à 4 mm do limbo \\
\hline $\mathbf{1 0}$ & Tração conjuntival com cotonete \\
\hline $\mathbf{1 1}$ & $\begin{array}{l}\text { Aplicação de injeção perpendicular à esclera, } \\
\text { em local previamente marcado, com inserção de } \\
\text { pelo menos } 6 \text { mm da agulha em direção posterior } \\
\text { (CUIDADO PARA NÃO TOCAR NA MARGEM }\end{array}$ \\
\hline $\mathbf{1 2}$ & $\begin{array}{l}\text { Retirada da agulha e leve compressão } \\
\text { local com cotonete }\end{array}$ \\
\hline $\mathbf{1 3}$ & $\begin{array}{l}\text { Instilação de colírio de antibiótico associado a } \\
\text { corticóide ou PVPI }\end{array}$ \\
\hline $\mathbf{1 4}$ & $\begin{array}{l}\text { Retirada de blefarostato e campo oftálmico } \\
\text { fenestrado estéril }\end{array}$ \\
\hline
\end{tabular}

Após ponderação de risco $\mathrm{x}$ benefício no que diz respeito à nossa população de pacientes, optamos por ainda não instituir injeções bilaterais no mesmo dia como prática rotineira, apesar das evidências favoráveis à sua realização. Uma potencial limitação para essa prática em nosso serviço seria a logística na utilização de lotes diferentes de BEZ para cada olho em um mesmo paciente.

Os benefícios dessa prática, como melhor adesão e comodidade por parte do paciente e utilização mais eficiente das vagas disponíveis para o procedimento, devem fomentar maiores discussões quanto a instauração dessa medida no futuro.

Os estudos demonstraram segurança com o uso de BEZ fracionado, condizente com nossa estatística de 3 casos de endoftalmite infecciosa em períodos temporais distintos na trajetória de 10 anos em que realizamos aproximadamente 10.000 injeções intravítreas $(0.03 \%)$, valor tangenciando a margem inferior da incidência relatada na literatura $(0.028 \% \text { a } 0.056 \%)^{12,13}$.

Algumas medidas com evidência baixa mas bem estabelecidas na realização de procedimentos em geral e rotinas na nossa prática são o uso de luvas e máscaras, assim como higienização das mãos. A restrição à conversa na sala cirúrgica possui baixa evidência para sua realização, mas é de fácil instituição e com benefícios teóricos.

Apesar de disponível em nosso serviço, o uso de campo cirúrgico estéril adesivo acrescentaria custo para a instituição e desconforto para os pacientes, sem relato de benefícios claros na literatura, portanto utilizamos um campo oftálmico estéril convencional. Utilizamos o blefarostato de Barraquer como método de afastamento palpebral.

O uso de PVPI 5\% tópico pré operatório é a medida com maior evidência para profilaxia de endoftalmite e é realizada de forma rotineira em nosso serviço. Alguns aspectos que não haviam sido contemplados são a aplicação do PVPI após colocação o blefarostato e uma segunda aplicação, que foram instituídos após essa revisão.

Quanto ao material recomendado, atualmente temos disponíveis apenas agulhas com calibre de 26.5 gauge para o procedimento, com maior risco de refluxo e maior desconforto para nossos pacientes. Está em andamento o processo para obtenção de agulhas 30 gauge.

As evidências não embasam o uso de antibióticos tópicos pré e pós operatórios de forma profilática, com grau razoável de evidência. Optamos por manter a aplicação de uma gota de ofloxacina ou PVPI $5 \%$ tópicos logo após o término do procedimento, de forma similar à rotina de outras intervenções intraoculares mais invasivas, tendo em vista o perfil risco x benefício favorável. Não indicamos, porém, uso de antibióticos fora do período transoperatório.

Finalmente, as medidas que estabelecemos estão condizentes com os principais guidelines e estudos disponíveis sobre o assunto, visando a melhoria da qualidade assistencial em nosso serviço. Os pontos discordantes serão alvo de estudo em busca da 
otimização, dentro do contexto de serviço público de saúde e levando em consideração aspectos de custo-efetividade.

\section{Conflitos de Interesse}

Os autores declaram não haver conflito de interesses.

\section{REFERÊNCIAS}

1. Peyman GA, Lad EM, Moshfeghi DM. Intravitreal injection of therapeutic agents. Retina. 2009;29(7):875-912.

2. McLaughlin MD, Hwang JC. Trends in vitreoretinal procedures for medicare beneficiaries, 2000 to 2014 Ophthalmology. 2017;124(5):667-73.

3. Lee SS, Hughes PM, Robinson MR. Recent advances in drug delivery systems for treating ocular complications of systemic diseases. Curr Opin Ophthalmol. 2009;20(6):511-9.

4. Klaassen I, Van Noorden CJ, Schlingemann RO. Molecular basis of the inner blood-retinal barrier and its breakdown in diabetic macular edema and other pathological conditions. Prog Retin Eye Res. 2013;34:19-48.

5. Tang J, Kern TS. Inflammation in diabetic retinopathy. Prog Retin Eye Res. 2011;30(5):343-58.

6. Mallmann F, Canani LH. Intravitreal neurodegenerative and inflammatory mediators in proliferative diabetic retinopathy. Arq Bras Oftalmol. 2019;82(4):275-82.

7. Varma R, Bressler NM, Suñer I, Lee P, Dolan CM, Ward J, et al. Improved vision-related function after ranibizumab for macular edema after retinal vein occlusion: results from the BRAVO and CRUISE trials. Ophthalmology. 2012;119(10):2108-18.

8. Fardeau C, Champion E, Massamba N, LeHoang P. Uveitic macular edema. Eye (Lond). 2016;30(10):1277-92.

9. Nguyen QD, Brown DM, Marcus DM, Boyer DS, Patel S, Feiner L, et al. Ranibizumab for diabetic macular edema: results from 2 phase III randomized trials: RISE and RIDE. Ophthalmology. 2012;119(4):789-801.

10. Hilton GF, Kelly NE, Salzano TC, Tornambe PE, Wells JW, Wendel RT. Pneumatic retinopexy. A collaborative report of the first 100 cases. Ophthalmology. 1987;94(4):307-14.

11. Relhan N, Forster RK, Flynn HW. Endophthalmitis: then and now. Am J Ophthalmol. 2018;187:10-7.
12. Fileta JB, Scott IU, Flynn HW. Metaanalysis of infectious endophthalmitis after intravitreal injection of antivascular endothelial growth factor agents. Ophthalmic Surg Lasers Imaging Retina. 2014;45(2):143-9.

13. Merani R, Hunyor AP. Endophthalmitis following intravitreal anti-vascular endothelial growth factor (VEGF) injection: a comprehensive review. Int J Retina Vitreous. 2015;1:9.

14. Avery RL, Bakri SJ, Blumenkranz MS, Brucker AJ, Cunningham ET Jr, D'Amico DJ, et al. Intravitreal injection technique and monitoring: updated guidelines of an expert panel. Retina. 2014;34(Suppl 12):S1-S18.

15. Grzybowski A, Told R, Sacu S Bandello F, Moisseiev E, Loewenstein A, et al. 2018 update on intravitreal injections: euretina expert consensus recommendations. Ophthalmologica. 2018;239(4):181-93.

16. Lyall DAM, Tey A, Foot B, Roxburgh STD, Virdi M, Robertson C, et al. Post-intravitreal anti-VEGF endophthalmitis in the United Kingdom: incidence, features, risk factors, and outcomes. Eye (Lond). 2012;26(12):1517-26.

17. Lemos V, Cabugueira A, Noronha M, Pinto LA, Reina M, Branco J, et al. Intraocular pressure in eyes receiving intravitreal antivascular endothelial growth factor injections. Ophthalmologica. 2015;233(3-4):162-8.

18. Hollands $\mathrm{H}$, Wong J, Bruen R, Campbell RJ, Sharma S, Gale J. Short-term intraocular pressure changes after intravitreal injection of bevacizumab. Can J Ophthalmol. 2007;42(6):807-11.

19. Mojica G, Hariprasad SM, Jager RD, Mieler WF. Short-term intraocular pressure trends following intravitreal injections of ranibizumab (Lucentis) for the treatment of wet age-related macular degeneration. Br J Ophthalmol. 2008;92(4):584.

20. Gismondi M, Salati C, Salvetat ML, Zeppieri M, Brusini P. Short-term effect of intravitreal injection of Ranibizumab (Lucentis) on intraocular pressure. J Glaucoma. 2009;18(9):658-61.
21. Yannuzzi NA, Patel SN, Bhavsar KV, Sugiguchi F, Freund KB. Predictors of sustained intraocular pressure elevation in eyes receiving intravitreal anti-vascular endothelial growth factor therapy. Am J Ophthalmol. 2014;158(2):319-27.

22. Tranos P, Bhar G, Little B. Postoperative intraocular pressure spikes: the need to treat. Eye (Lond). 2004;18(7):673-9.

23. Ozcaliskan S, Ozturk F, Yilmazbas P, Beyazyildiz O. Effect of dorzolamidetimolol fixed combination prophylaxis on intraocular pressure spikes after intravitreal bevacizumab injection. Int J Ophthalmol. 2015;8(3):496-500.

24. Frenkel MPC, Haji SA, Frenkel REP. Effect of prophylactic intraocular pressure-lowering medication on intraocular pressure spikes after intravitreal injections. Arch Ophthalmol. 2010;128(12):1523-7.

25. Yuksel-Elgin C, Elgin C. Intraocular pressure elevation after intravitreal triamcinolone acetonide injection: a meta-analysis. Int $J$ Ophthalmol. 2016;9(1):139-44.

26. Dedania VS, Bakri SJ. Sustained elevation of intraocular pressure after intravitreal anti-vegf agents: what is the evidence? Retina. 2015;35(5):841-58.

27. Beck RW, Edwards AR, Aiello LP, Bressler NM, Ferris F, Glassman AR, et al. Three-year followup of a randomized trial comparing focal/grid photocoagulation and intravitreal triamcinolone for diabetic macular edema. Arch Ophthalmol. 2009;127(3):245-51.

28. Scappaticci FA, Skillings JR, Holden SN, Gerber HP, Miller K, Kabbinavar F, et al. Arterial thromboembolic events in patients with metastatic carcinoma treated with chemotherapy and bevacizumab. $J$ Natl Cancer Inst. 2007;99(16):1232-9.

29. Hapani S, Sher A, Chu D, Wu S. Increased risk of serious hemorrhage with bevacizumab in cancer patients: a meta-analysis. Oncology. 2010;79(1-2):27-38. 
30. Zehetner C, Kirchmair R, Huber S, Kralinger MT, Kieselbach GF. Plasma levels of vascular endothelial growth factor before and after intravitreal injection of bevacizumab, ranibizumab and pegaptanib in patients with age-related macular degeneration, and in patients with diabetic macular oedema. Br J Ophthalmol. 2013;97(4):454-9.

31. Thulliez M, Angoulvant D, Pisella PJ, Bejan-Angoulvant T. Overview of systematic reviews and metaanalyses on systemic adverse events associated with intravitreal antivascular endothelial growth factor medication use. JAMA Ophthalmol. 2018;136(5):557-66.

32. Olson JM, Scott IU, Kerchner DL, Kunselman AR. Association between systemic anticoagulation and rate of intraocular hemorrhage following intravitreal anti-VEGF therapy for age-related macular degeneration. Ophthalmic Surg Lasers Imaging Retina. 2013;44(5):455-9.

33. Veritti D, Di Giulio A, Sarao V, Lanzetta P. Drug safety evaluation of intravitreal triamcinolone acetonide. Expert Opin Drug Saf. 2012;11(2):331-40.

34. Fazelat A, Lashkari K. Off-label use of intravitreal triamcinolone acetonide for diabetic macular edema in a pregnant patient. Clin Ophthalmol. 2011;5:439-41.

35. Introini U, Casalino G, Cardani A, Scotti F, Finardi A, Candiani M, et al. Intravitreal bevacizumab for a subfoveal myopic choroidal neovascularization in the first trimester of pregnancy. J Ocul Pharmacol Ther. 2012;28(5):553-5.

36. Polizzi S, Mahajan VB. Intravitreal anti-VEGF injections in pregnancy: case series and review of literature. J Ocul Pharmacol Ther. 2015;31(10):605-10.

37. McFarland TJ, Rhoads AD, Hartzell M, Emerson GG, Bhavsar AR, Stout JT. Bevacizumab levels in breast milk after long-term intravitreal injections. Retina. 2015;35(8):1670-3.

38. Patel SN, Gangaputra S, Sternberg P Jr, Kim SJ. Prophylaxis measures for postinjection endophthalmitis. Surv Ophthalmol. 2020;65(4):408-20.
39. Xing L, Dorrepaal SJ, Gale J. Survey of intravitreal injection techniques and treatment protocols among retina specialists in Canada. Can J Ophthalmol. 2014;49(3):261-6.

40. Rayess N, Rahimy E, Storey P, Shah CP, Wolfe JD, Chen E, et al. Postinjection endophthalmitis rates and characteristics following intravitreal bevacizumab, ranibizumab, and aflibercept. Am J Ophthalmol. 2016;165:88-93.

41. Wani VB, Al-Kandari J, Sabti K, Aljassar F, Qali H, Kumar N, et al. Incidence of endophthalmitis after intravitreal bevacizumab using aliquots prepared on-site in 2 operating rooms in Kuwait. Middle East Afr J Ophthalmol. 2016;23(1):64-70.

42. Casparis $\mathrm{H}$, Wolfensberger $\mathrm{TJ}$, Becker M, Eich G, Graf N, Ambresin A, et al. Incidence of presumed endophthalmitis after intravitreal injection performed in the operating room: a retrospective multicenter study. Retina. 2014;34(1):12-7.

43. McCannel CA. Meta-analysis of endophthalmitis after intravitreal injection of anti-vascular endothelial growth factor agents: causative organisms and possible prevention strategies. Retina. 2011;31(4):654-61.

44. Wen JC, McCannel CA, Mochon AB, Garner OB. Bacterial dispersal associated with speech in the setting of intravitreous injections. Arch Ophthalmol. 2011;129(12):1551-4.

45. O'Grady NP, Alexander M, Dellinger EP, Gerberding JL, Heard SO, Maki DG, et al. Guidelines for the prevention of intravascular catheter-related infections. MMWR Recomm Rep. 2002;51(RR-10):1-29.

46. Garg SJ, Dollin M, Hsu J, Storey P, Vander JF. Effect of a strict 'no-talking' policy during intravitreal injection on post-injection endophthalmitis. Ophthalmic Surg Lasers Imaging Retina. 2015;46(10):1028-34.

47. Stem MS, Rao P, Lee IJ, Woodward MA, Faia LJ, Wolfe JD, et al. Predictors of endophthalmitis after intravitreal injection: a multivariable analysis based on injection protocol and povidone iodine strength. Ophthalmol Retina. 2019;3(1):3-7.

48. Bhavsar AR, Googe JM, Stockdale CR, Bressler NM, Brucker AJ, Elman MJ, et al. Risk of endophthalmitis after intravitreal drug injection when topical antibiotics are not required: the diabetic retinopathy clinical research network laser-ranibizumab-triamcinolone clinical trials. Arch Ophthalmol. 2009;127(12):1581-3.

49. Tailor R, Beasley R, Yang Y, Narendran N. Evaluation of patients' experiences at different stages of the intravitreal injection procedure - what can be improved? Clin Ophthalmol. 2011;5:1499-502.

50. Mansour AM, Shahin M, Kofoed PK, Parodi MB, Shami M, Schwartz SG, et al. Insight into 144 patients with ocular vascular events during VEGF antagonist injections. Clin Ophthalmol. 2012;6:343-63.

51. Shah CP, Garg SJ, Vander JF, Brown GC, Kaiser RS, Haller JA, et al. Outcomes and risk factors associated with endophthalmitis after intravitreal injection of anti-vascular endothelial growth factor agents. Ophthalmology. 2011;118(10):2028-34.

52. Munro M, Williams GR, Ells A, Fielden M, Kherani A, Mitchell P, et al. Lid splinting eyelid retraction technique: a minimised sterile approach for intravitreal injections. Br J Ophthalmol. 2018;102(9):1254-8.

53. Chaturvedi R, Wannamaker KW, Riviere PJ, Khanani AM, Wykoff CC, Chao DL. Real-world trends in intravitreal injection practices among American retina specialists. Ophthalmol Retina. 2019;3(8):656-62.

54. Doshi RR, Leng T, Fung AE. Povidone-iodine before lidocaine gel anesthesia achieves surface antisepsis. Ophthalmic Surg Lasers Imaging. 2011;42(4):346-9.

55. Speaker MG, Milch FA, Shah MK, Eisner W, Kreiswirth BN. Role of external bacterial flora in the pathogenesis of acute postoperative endophthalmitis. Ophthalmology. 1991;98(5):639-49.

56. Ciulla TA, Starr MB, Masket S. Bacterial endophthalmitis prophylaxis for cataract surgery: an evidencebased update. Ophthalmology. 2002;109(1):13-24.

57. Bigliardi PL, Alsagoff SAL, El-Kafrawi HY, Pyon JK, Wa CTC, Villa MA. Povidone iodine in wound healing: a review of current concepts and practices. Int J Surg. 2017;44:260-8. 
58. Friedman DA, Mason JO, Emond T, McGwin G. Povidone-iodine contact time and lid speculum use during intravitreal injection. Retina. 2013;33(5):975-81.

59. Stranz CV, Fraenkel GE, Butcher AR, Esterman AJ, Goggin MJ. Survival of bacteria on the ocular surface following double application of povidone-iodine before cataract surgery. Eye (Lond). 2011;25(11):1423-8.

60. Levinson JD, Garfinkel RA, Berinstein DM, Flory M, Spellman FA. Timing of povidone-iodine application to reduce the risk of endophthalmitis after intravitreal injections. Ophthalmol Retina. 2018;2(7):654-8.

61. Lachapelle JM. A comparison of the irritant and allergenic properties of antiseptics. Eur J Dermatol. 2014;24(1):3-9.

62. Krohne TU, Allam JP, Novak N, Holz FG. "lodine allergy": A medical myth with risks for the ophthalmological patient. Ophthalmologe. 2016;113(12):1023-8.

63. Grzybowski A, Kanclerz P, Myers WG. The use of povidone-iodine in ophthalmology. Curr Opin Ophthalmol. 2018;29(1):19-32.

64. Wykoff CC, Flynn HW, Han DP. Allergy to povidone-iodine and cephalosporins: the clinical dilemma in ophthalmic use. Am J Ophthalmol. 2011;151(1):4-6.

65. Schabelman E, Witting M. The relationship of radiocontrast, iodine, and seafood allergies: a medical myth exposed. J Emerg Med. 2010;39(5):701-7.
66. Merani R, McPherson ZE, Luckie AP, Gilhotra JS, Runciman J, Durkin S, et al. Aqueous chlorhexidine for intravitreal injection antisepsis: a case series and review of the literature. Ophthalmology. 2016;123(12):2588-94.

67. Borkar DS, Obeid A, Su DC, Storey PP, Gao X, Regillo CD, et al. Endophthalmitis rates after bilateral same-day intravitreal anti-vascular endothelial growth factor injections. Am J Ophthalmol. 2018;194:1-6.

68. Rifkin L, Schaal S. Factors affecting patients' pain intensity during in office intravitreal injection procedure. Retina. 2012;32(4):696-700.

69. Hubschman JP, Coffee RE, Bourges JL, Yu F, Schwartz SD. Experimental model of intravitreal injection techniques. Retina. 2010;30(1):167-73.

70. Karimi S, Mosavi SA, Jadidi K, Nikkhah $\mathrm{H}$, Kheiri B. Which quadrant is less painful for intravitreal injection? A prospective study. Eye (Lond). 2019;33(2):304-12.

71. d'Azy CB, Pereira B, Naughton G, Chiambaretta F, Dutheil F. Antibioprophylaxis in prevention of endophthalmitis in intravitreal injection: a systematic review and metaanalysis. PLoS One. 2016;11(6):1-12.

72. Schwartz SG, Flynn HW, Grzybowski A. Controversies in topical antibiotics use with intravitreal injections. Curr Pharm Des. 2015;21(32):4703-6.

73. Bhavsar AR, Stockdale CR, Ferris FL, Brucker AJ, Bressler NM, Glassman AR, et al. Update on risk of endophthalmitis after intravitreal drug injections and potential impact of elimination of topical antibiotics. Arch Ophthalmol. 2012;130(6):809-10.
74. Goldberg RA, Flynn HW, Isom RF, Miller D, Gonzalez S. An outbreak of streptococcus endophthalmitis after intravitreal injection of bevacizumab. Am J Ophthalmol. 2012;153(2):204-8.

75. Yannuzzi NA, Klufas MA, Quach L, Beatty LM, Kaminsky SM, Crystal RG, et al. Evaluation of compounded bevacizumab prepared for intravitreal injection. JAMA Ophthalmol. 2015;133(1):32-9.

76. Tabatabaii A, Ahmadraji A, Khodabande A, Mansouri M. Acute bilateral endophthalmitis following bilateral intravitreal bevacizumab (avastin) injection. Middle East Afr J Ophthalmol. 2013;20(1):87-8.

77. Ladas ID, Karagiannis DA, Rouvas AA, Kotsolis Al, Liotsou A, Vergados I. Safety of repeat intravitreal injections of bevacizumab versus ranibizumab: our experience after 2,000 injections. Retina. 2009;29(3):313-8.

78. Falavarjani KG, Nguyen QD. Adverse events and complications associated with intravitreal injection of anti-VEGF agents: a review of literature. Eye (Lond). 2013;27(7):787-94.

79. Filho PA, Maia M, Rodrigues EB, Farah ME. Ocular pharmacology in the treatment of vitreous, retina and choroid diseases. Arq Bras Oftalmol. 2010;73(3):294-9.

80. Rodrigues EB, Maia M, Penha FM, Dib E, Bordon AF, Magalhães Júnior $\mathrm{O}$, et al. Technique of intravitreal drug injection for therapy of vitreoretinal diseases. Arq Bras Oftalmol. 2008;71(6):902-7.

81. Sampat KM, Garg SJ. Complications of intravitreal injections. Curr Opin Ophthalmol. 2010;21(3):178-83.

Recebido: 17 jul, 2020

Aceito: 19 out, 2020 